\title{
An unusual cause of a limp in a child: developmental coxa vara
}

\author{
F Lam, S Hussain, J Sinha
}

A 3 year old girl of Afro-Caribbean origin presented to the accident and emergency department with a two week history of right sided hip pain and limp. There was no history of trauma and she was otherwise well with no systemic symptoms. On examination, she walked with a Trendelenburg gait and her right leg was noted to be shortened and externally rotated at rest. The range of movements in her right hip was reduced especially abduction and internal rotation. Otherwise, physical examination was unremarkable. Pelvic radiographs were taken showing gross postero-inferior displacement of the right upper femoral epiphysis, decreased femoral neck shaft angle and a triangular metaphyseal fragment in the inferior femoral neck (fig 1). These are all classic radiological features of developmental coxa vara.

Coxa vara is a varus deformity of the femoral neck with a decrease in the angle between the neck and shaft of femur, which is normally between $135-145^{\circ}$ in children. It may be congenital, developmental in which symptoms appear during early childhood when walking is started or acquired secondary to an underlying metabolic, neoplastic or traumatic cause. Developmental coxa vara is a rare condition with an incidence of 1 in 25000 live births, 20 times rarer than congenital hip dislocation. ${ }^{1}$ It has a preponderance in black people and its natural history if untreated is one of progressive varus deformity of the proximal femur that can ultimately lead to stress fracture related non-union of the femoral neck, pseudarthrosis formation and premature degenerative changes within the hip joint. ${ }^{2}$ Treatment is in the form of a valgus producing trochanteric osteotomy.

This case illustrates several important learnTrauma and Orthopaedic Surgery, King's College Hospital, Denmark Hill, London SE5 9RS, UK ing points. Firstly, a detailed history and a careful examination is mandatory in the evaluation of hip pain and limp in a child. To the inexperienced, the radiological features of coxa vara may sometimes be misinterpreted as a

Correspondence to: Mr Lam

(mrflam@hotmail.com)

Accepted for publication 25 January 2001 traumatic fracture as it did in our case. Furthermore, in the absence of a positive history of trauma volunteered by the parents, the issue of non-accidental injury may be
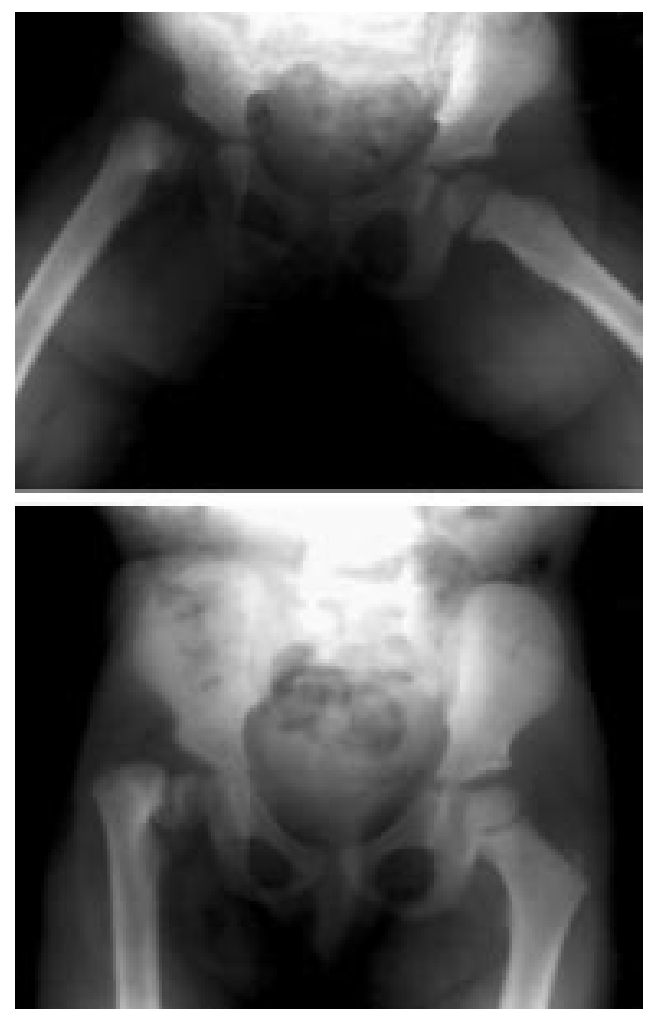

Figure 1 AP and lateral pelvic radiographs showing a decreased femoral neck shaft angle, triangular fragment in the inferior femoral neck and the appearance of the upper femoral epiphysis slipping off the femoral neck.

wrongly suspected. Secondly, early diagnosis of the condition is important as $83 \%$ of patients achieve excellent functional results in terms of acetabular depth, spherical congruency, relief from pain, and correction of gait if the varus deformity can be corrected and maintained before age $10 .^{3}$

1 Johanning K. Coxa vara infantum. Acta Orthop Scand 1951;21:273.

2 Serafin J, Szule W. Coxa vara infantum, hip growth disturbances, etiopathogenesis, and long term results of disturbances, etiopathogenesis, and long

3 Carroll K, Coleman S, Stevens PM. Coxa vara: surgical outcomes of valgus osteotomies. F Pediatr Orthop 1997;17: 220-4. 